\title{
ON A NEW FIXED POINT THEOREM ON HILBERT SPACES VIA THE MOUNTAIN PASS LEMMA AND APPLICATIONS TO ELLIPTIC BOUNDARY VALUE PROBLEMS
}

\author{
Ayadi Souad $^{1}$, Toufik Moussaoui ${ }^{2}$, Donal O'Regan $^{3}$ \\ ${ }^{1}$ Laboratory of Physical Mechanics and Mathematical Modeling \\ Medea, ALGERIA \\ ${ }^{1}$ Laboratory of Fixed Point Theory and Applications \\ Department of Mathematics \\ E.N.S. Kouba, Algiers, ALGERIA \\ ${ }^{2}$ Laboratory of Fixed Point Theory and Applications \\ Department of Mathematics \\ E.N.S. Kouba, Algiers, ALGERIA \\ ${ }^{3}$ School of Mathematics, Statistics and Applied Mathematics \\ National University of Ireland \\ Galway, IRELAND
}

\begin{abstract}
In this paper we present a new fixed point theorem in Hilbert spaces. Using a suitable critical point theorem we provide conditions under which potential operators have a fixed point. An application is given to illustrate the theory.
\end{abstract}

Key Words: fixed point theorem, potential operators

\section{Introduction}

Let $(H,\langle.,\rangle$.$) be a real Hilbert space and let \langle.,$.$\rangle denote the scalar product on$

Received: January 18, 2016

Published: February 3, 2016 (c) 2016 Academic Publications, Ltd.

url: www.acadpubl.eu 
$H$ and $\|\|=.\sqrt{\langle., .\rangle}$ the norm. A functional $\varphi: H \longrightarrow \mathbb{R}$ is said to be Gâteaux differentiable on $H$ if

$$
\lim _{t \rightarrow 0} \frac{\varphi(u+t h)-\varphi(u)}{t}=(\operatorname{Grad} \varphi(u), h) \quad \forall u, h \in H .
$$

The operator $T: H \longrightarrow H$, is called a potential operator (or a gradient operator) on $H$, if there exists a Gâteaux differentiable functional $\varphi$ such that $\operatorname{Grad} \varphi(u)=T(u)$, for all $u \in H$.

An operator $T: H \longrightarrow H$ is said to have a fixed point in $H$ if there exists $u_{0} \in H$ such that $T\left(u_{0}\right)=u_{0}$. In [6] the authors rephrase the problem of searching for fixed points of a potential operator $T$ to searching for critical points of the functional $J$ with $J^{\prime}=I-T$. Recall that a critical point for $J$ on $H$ is an element $u_{0} \in H$ such that, $J^{\prime}\left(u_{0}\right)=0$. We give conditions on the potential operator $T$ so that the Mountain Pass Theorem guarantees the existence of critical points of $J$, so as a result guarantees the existence of fixed points of $T$. First, we recall some concepts from critical point theory.

Definition 1.1. $[2,1,3]$ Let $J \in C^{1}(H, \mathbb{R})$. If any sequence $\left(u_{n}\right) \subset H$ for which $\left(J\left(u_{n}\right)\right)$ is bounded in $\mathbb{R}$ and $J^{\prime}\left(u_{n}\right) \longrightarrow 0$ as $n \rightarrow+\infty$ in $H$ possesses a convergent subsequence, then we say that $J$ satisfies the Palais-Smale condition (PS condition for short).

Theorem 1.1. (Mountain Pass Theorem [5, 3]) Let $H$ be a Banach space and let $J \in C^{1}(H, \mathbb{R})$, satisfy the Palais-Smale condition. Assume $J(0)=0$ and there exist positive numbers $\rho$, and $\alpha$ such that:

1. $J(u) \geq \alpha$ if $\|u\|=\rho$,

2. There exists $\vartheta \in H$ such that $\|\vartheta\|>\rho$ and $J(\vartheta)<\alpha$.

Then $c$ is a critical value of $J$ with $c \geq \alpha$, where $c=\min _{A \in \Gamma} \max _{u \in A} J(u)$, and $\Gamma=\{\gamma([0,1]) ; \gamma \in C([0,1], H) \gamma(0)=0, \gamma(1)=\vartheta\}$.

Theorem 1.2. [1] Let $H$ and $Y$ be two Banach spaces, $\Omega$ an open subset of $H$, and $\varphi: \Omega \longrightarrow Y$ a mapping of class $C^{1}$. Given $x, y \in \Omega$, if $x+$ ty $\in \Omega$ for all $t \in(0,1)$, then

$$
\varphi(x+y)=\varphi(x)+\int_{0}^{1}<D \varphi(x+t y), y>d t .
$$

This result connects the potential operator $T$ and the Gâteaux differentiable functional $\varphi$ since

$$
\varphi(x)=\int_{0}^{1}(T(s x), x) d s .
$$


Following the ideas in [6], the authors in [7] used a suitable minimization principle to prove the following fixed point theorem

Theorem 1.3. Let $H$ be a Hilbert space and $T: H \longrightarrow H$ a compact potential operator such that there exists a bounded linear operator $B$ on $H$ and $v^{*} \in H$ satisfying

$$
(T(s u), u) \leq(B(s u), u)+\left(v^{*}, u\right), \forall s \in[0,1], \forall u \in H \text { with }\|B\|<1 .
$$

Then, the operator $T$ has a fixed point in $H$.

In this paper we prove a new fixed point theorem which weakens the condition in the above theorem.

\section{Existence Result}

Theorem 2.1. Let $H$ be a Hilbert space and $T: H \longrightarrow H$ a compact potential operator such that there exists a bounded linear operator $B$ on $H$ and a $v^{*} \in H^{\prime}$ satisfying

1. $(T(s u), u) \leq(B(s u), u)+\left\langle v^{*}, u\right\rangle, \forall s \in(0,1), \forall u \in H \quad$ with $\|u\|=$ $\rho_{0}$, for some $\rho_{0}>\frac{2\left\|v^{*}\right\|}{1-\|B\|},\|B\|<1$,

2. $\exists e \in H$ with $\|e\|=1$ and $(B(e), e)>1$ such that, $(T(s e), e) \geq(B(s e), e), \quad \forall s \in(0,1)$,

Then, the operator $T$ has a fixed point in $H$.

Proof. Since $T$ is a potential operator, there exists a Gâteaux differentiable functional $\varphi: H \longrightarrow \mathbb{R}$ such that $\varphi^{\prime}=T$. Let $J=I-\varphi$. Then $J \in C^{1}(H, \mathbb{R})$ with $J^{\prime}=I-\varphi^{\prime}=I-T$, i.e., $\forall u \in H, J^{\prime}(u)=u-T u$. Since $T$ is a potential operator, it can be represented in the form

$$
\varphi(u)=\int_{0}^{1}(T(s u), u) d s .
$$

Consider the functional $J: H \longrightarrow \mathbb{R}$ defined by

$$
J(u)=\frac{1}{2}\|u\|^{2}-\int_{0}^{1}(T(s u), u) d s .
$$


Step 1. $J$ satisfies the Palais-Smale condition.

Let $\left(u_{n}\right)$ be a sequence in $H$ such that $\lim _{n \rightarrow+\infty} J^{\prime}\left(u_{n}\right)=0$ and $\left(J\left(u_{n}\right)\right)$ is bounded. i.e., there exists some positive constant $K$ such that $\left|J\left(u_{n}\right)\right| \leq K$, for all positive integer $n$. From assumption (1), we have

$$
\begin{aligned}
K \geq J\left(u_{n}\right) & \geq \frac{1}{2}\left\|u_{n}\right\|^{2}-\int_{0}^{1}\left[\left(B\left(s u_{n}\right), u_{n}\right)+\left\langle v^{*}, u_{n}\right\rangle\right] d s \\
& \geq \frac{1}{2}\left\|u_{n}\right\|^{2}-\int_{0}^{1} s\left\|u_{n}\right\|^{2}\|B\| d s-\int_{0}^{1}\left\langle v^{*}, u_{n}\right\rangle d s \\
& \geq \frac{1}{2}\left\|u_{n}\right\|^{2}-\frac{1}{2}\left\|u_{n}\right\|^{2}\|B\|-\left\|v^{*}\right\|\left\|u_{n}\right\| \\
& =\frac{1}{2}(1-\|B\|)\left\|u_{n}\right\|^{2}-\left\|v^{*}\right\|\left\|u_{n}\right\|,
\end{aligned}
$$

which implies that $\left(u_{n}\right)$ is bounded in $H$. We note that, $J^{\prime}\left(u_{n}\right)=u_{n}-T\left(u_{n}\right)$, with $\lim _{n \rightarrow+\infty} J^{\prime}\left(u_{n}\right)=0$. Since the sequence $\left(u_{n}\right)$ is bounded and the operator $T$ is compact, the sequence $\left(T\left(u_{n}\right)\right)$ is relatively compact, and as a consequence there exists a subsequence $\left(u_{n_{k}}\right) \subset\left(u_{n}\right)$ such that $T\left(u_{n_{k}}\right) \longrightarrow w$. Hence $u_{n_{k}} \longrightarrow w$ in $H$ (note

$$
\left.\left\|u_{n_{k}}-w\right\| \leq\left\|u_{n_{k}}-T\left(u_{n_{k}}\right)\right\|+\left\|T\left(u_{n_{k}}\right)-w\right\| \longrightarrow 0, \text { as } k \rightarrow+\infty\right) .
$$

Thus, the (PS) condition is satisfied.

Step 2. $J$ satisfies assumption (1) of Theorem 1.1.

Consider any $\rho_{0}>\frac{2\left\|v^{*}\right\|}{1-\|B\|}$, and let $\alpha_{0}=\frac{1}{2} \rho_{0}\left((1-\|B\|) \rho_{0}-2\left\|v^{*}\right\|\right)$. From assumption (1), if $\|u\|=\rho_{0}$ we have

$$
\begin{aligned}
J(u) & \geq \frac{1}{2}\|u\|^{2}-\int_{0}^{1}\left[(B(s u), u)+\left\langle v^{*}, u\right\rangle\right] d s \\
& \geq \frac{1}{2}\|u\|^{2}-\int_{0}^{1} s\|u\|^{2}\|B\| d s-\int_{0}^{1}\left\langle v^{*}, u\right\rangle d s \\
& \geq \frac{1}{2}\|u\|^{2}-\frac{1}{2}\|u\|^{2}\|B\|-\left\|v^{*}\right\|\|u\| \\
& =\frac{1}{2}(1-\|B\|)\|u\|^{2}-\left\|v^{*}\right\|\|u\| \\
& =\frac{1}{2}\|u\|\left((1-\|B\|)\|u\|-2\left\|v^{*}\right\|\right) \\
& =\frac{1}{2} \rho_{0}\left((1-\|B\|) \rho_{0}-2\left\|v^{*}\right\|\right)
\end{aligned}
$$




$$
=\alpha_{0}
$$

Step 3. $J$ satisfies assumption (2) of Theorem 1.1.

From assumption (2), there exists $e \in H$ such that,

$$
\|e\|=1 \text { and } 1-(B(e), e)<0 .
$$

Let $\vartheta=\xi e$, with $\xi>0$. Then

$$
\begin{aligned}
J(\xi e) & =\frac{1}{2}\|\xi e\|^{2}-\int_{0}^{1}(T(s \xi e), \xi e) d x \\
& \leq \frac{1}{2}\|\xi e\|^{2}-\int_{0}^{1}(B(s \xi e), \xi e) d s \\
& =\frac{1}{2} \xi^{2}\left(\|e\|^{2}-(B(e), e)\right) \\
& =\frac{1}{2} \xi^{2}(1-(B(e), e)) \longrightarrow-\infty \text { as } \xi \longrightarrow+\infty
\end{aligned}
$$

The functional $J$ satisfies the hypotheses of the Mountain Pass Theorem, so $J$ has a critical point. Thus $T$ has a fixed point.

\section{Application}

Consider the problem

$$
\left\{\begin{array}{l}
-\Delta u=f(x, u), \quad x \in \Omega \\
u(x)=0, \quad x \in \partial \Omega
\end{array}\right.
$$

where $\Omega$ is a bounded domain in $\mathbb{R}^{N}, N \geq 1$, and $f: \Omega \times \mathbb{R} \longrightarrow \mathbb{R}$ a Carathéodory function satisfying the following growth condition:

there exist $a, b>0$ and $0 \leq \sigma \leq \frac{N+2}{N-2}$ if $N \geq 3(0 \leq \sigma<\infty$ if $N=1,2)$ such that

$$
|f(x, u)| \leq a|u|^{\sigma}+b, \forall x \in \Omega, \forall u \in \mathbb{R} .
$$

One can easily see that a weak solution of the problem (3.1) is a solution of the problem,

$$
\left\{\begin{array}{l}
\int_{\Omega} \nabla u \cdot \nabla v d x-\int_{\Omega} f(x, u(x)) \cdot v(x) d x=0, \quad \forall v \in H_{0}^{1}(\Omega), \\
u \in H_{0}^{1}(\Omega),
\end{array}\right.
$$


so, a weak solution of problem (3.1) satisfies

$$
(u, v)-\int_{\Omega} f(x, u(x)) \cdot v(x) d x=0, \quad \forall v \in H_{0}^{1}(\Omega) .
$$

Set $J^{\prime}(u) \cdot v=(u, v)-\int_{\Omega} f(x, u(x)) \cdot v(x) d x, \quad$ and let $\mathrm{T}$ be the operator defined by

$$
(T u, v)=\int_{\Omega} f(x, u(x)) \cdot v(x) d x, \forall v \in H_{0}^{1}(\Omega) .
$$

We have

$$
J^{\prime}(u) \cdot v=(u-T u, v)=((I-T) u, v), \text { and } T=I-J^{\prime}=(I-J)^{\prime}
$$

which implies that $T$ is a potential operator.

Lemma 1. ( [8], Remark 2.1)

The operator $T: H_{0}^{1}(\Omega) \longrightarrow H_{0}^{1}(\Omega)$ is compact.

Let $\lambda_{1}$ be the first eigenvalue of the linear Dirichlet problem

$$
\left\{\begin{array}{l}
-\Delta(x)=\lambda u(x), \quad x \in \Omega, \\
u(x)=0, \quad x \in \partial \Omega .
\end{array}\right.
$$

Recall $\|u\|_{\infty}=e s s \sup _{x \in \Omega}|u(x)|, \forall u \in L^{\infty}(\Omega)$.

Theorem 3.1. Assume the following conditions hold:

1. There exist functions $a, b \in L^{\infty}(\Omega)$ such that,

$$
\begin{aligned}
& \qquad f(x, u(x)) \leq a(x) u(x)+b(x), \forall x \in \Omega, \forall u \in L^{2}(\Omega) \text { with }\|u\|_{L^{2}} \leq r_{0}, \\
& \text { for some } r_{0}>\frac{1}{\sqrt{\lambda_{1}|\Omega|}} \text { and } 1-\frac{\|a\|_{\infty}}{\lambda_{1}}>2\|b\|_{\infty}|\Omega| \frac{1}{\sqrt{\lambda_{1}}} .
\end{aligned}
$$

2. There exists $w_{0} \in H_{0}^{1}$ with $\left\|w_{0}\right\|=1$, such that

$$
\begin{aligned}
& \frac{1}{\left\|w_{0}\right\|_{L^{2}}^{2}}<a(x)<\lambda_{1}, \quad \forall x \in \Omega \text { and } f\left(x, s w_{0}(x)\right) \geq a(x) s w_{0}(x), \quad \forall x \in \\
& \Omega, \forall s \in(0,1) .
\end{aligned}
$$

Then problem (3.1) has a weak solution.

Proof. We will apply Theorem 2.1. First we show assumption (1) of Theorem 2.1 is satisfied. Let $B$ be the bounded linear operator defined on $H_{0}^{1}(\Omega)$ by

$$
(B u, v)=\int_{\Omega} a(x) u(x) \cdot v(x) d x, \quad \forall u, v \in H_{0}^{1}(\Omega)
$$


and $v^{*} \in\left(H_{0}^{1}(\Omega)\right)^{\prime}$ be such that

$$
\left\langle v^{*}, v\right\rangle=\int_{\Omega} b(x) v(x) d x, \quad \forall v \in H_{0}^{1}(\Omega) .
$$

Let $\rho_{0}=r_{0} \sqrt{\lambda}_{1}$, and $u \in H_{0}^{1}(\Omega)$ such that $\|u\|=\rho_{0}$. Note $\|u\|_{L^{2}} \leq r_{0}$ and $\|s u\|_{L^{2}} \leq r_{0}$ for all $s \in(0,1)$. From assumption (1) of Theorem 3.1 we have

$$
\begin{aligned}
(T(s u), u) & =\int_{\Omega} f(x,(s u)(x)) \cdot u(x) d x \\
& \leq \int_{\Omega} a(x)(s u)(x) \cdot u(x)+\int_{\Omega} b(x) \cdot u(x) d x \\
& =(B(s u), u)+\left\langle v^{*}, u\right\rangle .
\end{aligned}
$$

Note (using the Poincaré inequality)

$$
\begin{aligned}
\|B u\| & =\sup _{\|v\| \leq 1}|\langle B u, v\rangle| \\
& =\sup _{\|v\| \leq 1}\left|\int_{0}^{1} a(x) u(x) v(x) d x\right| \\
& \leq\|a\|_{\infty} \sup _{\|v\| \leq 1} \int_{0}^{1}|u(x) v(x)| d x \\
& \leq\|a\|_{\infty} \sup _{\|v\| \leq 1}\|u\|_{L^{2}}\|v\|_{L^{2}} \\
& =\|a\|_{\infty}\|u\|_{L^{2}} \sup _{\|v\| \leq 1}\|v\|_{L^{2}} \\
& \leq\|a\|_{\infty} \frac{1}{\sqrt{\lambda_{1}}}\|u\| \sup _{\|v\| \leq 1} \frac{1}{\sqrt{\lambda_{1}}}\|v\| \\
& \leq \frac{1}{\lambda_{1}}\|a\|_{\infty}\|u\| .
\end{aligned}
$$

Since $B$ is a linear operator we have

$$
\|B\| \leq \frac{1}{\lambda_{1}}\|a\|_{\infty}<1
$$

Now we show $\rho_{0}>\frac{2\left\|v^{*}\right\|}{1-\|B\|}$. To see this notice (using the Cauchy-Schwarz and the Poincaré inequalities)

$$
\left\|v^{*}\right\|=\sup _{\|v\| \leq 1}\left|\left\langle v^{*}, v\right\rangle\right|
$$




$$
\begin{aligned}
& =\sup _{\|v\| \leq 1}\left|\int_{\Omega} b(x) v(x) d x\right| \\
& \leq\|b\|_{\infty} \sup _{\|v\| \leq 1} \int_{\Omega}|v(x)| d x \\
& \leq\|b\|_{\infty} \sup _{\|v\| \leq 1} \sqrt{|\Omega|}\|v\|_{L^{2}} \\
& =\|b\|_{\infty} \sqrt{|\Omega|} \sup _{\|v\| \leq 1}\|v\|_{L^{2}} \\
& \leq\|b\|_{\infty} \sqrt{|\Omega|} \frac{1}{\sqrt{\lambda_{1}}} \sup _{\|v\| \leq 1}\|v\| \\
& \leq\|b\|_{\infty} \sqrt{|\Omega|} \frac{1}{\sqrt{\lambda_{1}}} .
\end{aligned}
$$

Since $\frac{1}{\sqrt{\lambda_{1}}}<r_{0} \sqrt{|\Omega|}$ then $\left\|v^{*}\right\|<\|b\|_{\infty}|\Omega| r_{0}$ and so, $r_{0}>\frac{\left\|v^{*}\right\|}{\|b\|_{\infty}|\Omega|}$. Also since $\rho_{0}=r_{0} \sqrt{\lambda}_{1}$, we have

$$
\rho_{0}>\frac{\left\|v^{*}\right\| \sqrt{\lambda}_{1}}{\|b\|_{\infty}|\Omega|}
$$

Next since $\|B\| \leq \frac{\|a\|_{\infty}}{\lambda_{1}}$, then $1-\frac{\|a\|_{\infty}}{\lambda_{1}} \leq 1-\|B\|$. Also since $2\|b\|_{\infty}|\Omega| \frac{1}{\sqrt{\lambda}_{1}}<$ $1-\frac{\|a\|_{\infty}}{\lambda_{1}}$ one has

$$
2\|b\|_{\infty}|\Omega| \frac{1}{\sqrt{\lambda}_{1}}<1-\|B\|
$$

so $\frac{\sqrt{\lambda}_{1}}{2\|b\|_{\infty}|\Omega|}>\frac{1}{1-\|B\|}$ i.e. $\frac{2\left\|v^{*}\right\| \sqrt{\lambda_{1}}}{2\|b\|_{\infty}|\Omega|}>\frac{2\left\|v^{*}\right\|}{1-\|B\|}$, so $\rho_{0}>\frac{2\left\|v^{*}\right\|}{1-\|B\|}$.

Next we show assumption (2) of Theorem 2.1 is satisfied. First we show there exists $e \in H_{0}^{1}$ with $\|e\|=1$ such that $(B e, e)>1$. From assumption (2) of Theorem 3.1, there exists $w_{0} \in H_{0}^{1}(\Omega)$ with $\left\|w_{0}\right\|=1$, and $a(x)>$ $\frac{1}{\left\|w_{0}\right\|_{L^{2}}^{2}}, \forall x \in \Omega$. Put $e=w_{0}$ and note

$$
\begin{aligned}
(B e, e) & =\int_{\Omega} a(x)(e(x))^{2} d x \\
& >\frac{1}{\|e\|_{L^{2}}^{2}} \int_{\Omega}|e(x)|^{2} d x \\
& =1 .
\end{aligned}
$$


Next we show $(T(s e), e) \geq(B(s e), e)$ for all $s \in(0,1)$. From assumption (2) of Theorem 3.1, for $e=w_{0}$ we have $f(x, s e) \geq a(x)$ se, and so

$$
\begin{aligned}
(T(s e), e) & =\int_{\Omega} f(x, s e) \cdot e(x) d x, \quad \forall s \in(01) \\
& \geq \int_{\Omega} a(x)(s e)(x) \cdot e(x) d x \\
& =(B(s e), e) .
\end{aligned}
$$

From Theorem 2.1, the potential operator $T$ has a fixed point $u_{0}$ in $H_{0}^{1}(\Omega)$, which implies that $J^{\prime}\left(u_{0}\right)=0$. Thus problem (3.1) has a weak solution.

\section{References}

[1] C. Van Groesen, Variational Methods for Nonlinear Operator Equation, In Nonlinear Analysis: Proceeding of the lectures of a Colloquium Nonlinear Analysis. Mathematisch Centrum, Amsterdam, (1976), 100-191.

[2] J. Chabrowski, Variational Methods for Potential Operator Equation, Mathematisch Centrum, Amsterdam, 1979.

[3] O. Kavian, Introduction á la Théorie des Points Critiques et Applications aux Problèmes Elliptiques, Springer-Verlag, 1993.

[4] J. Mawhin, M. Willem, Critical Point Theory and Hamiltonian Systems. Springer-Verlag, Berlin (1989).

[5] M. Badiale, E. Serra, Semilinear Elliptic Equations for Beginners, Springer-Verlag, London, 2011.

[6] Y. Cui, J. Sun, Fixed point theorems for a class of nonlinear operators in Hilbert spaces and applications, Positivity, 15 (2011) 455-464.

[7] A. Boucenna, S. Djebali, and T. Moussaoui, Fixed point theorems for compact potential operators in Hilbert spaces, Submitted.

[8] D.G. Costa, An Invitation to Variational Methods in Differentiel Equations, Birkhauser, Boston, 2007. 
